\title{
Facile diazonium modification of pomegranate peel biochar: A stupendous derived relationship between thermal and Raman analyses
}

Authors:

Ahmed M. Khalil ${ }^{1,2,3, *}$, Radhia Msaadi ${ }^{4}$, Wafa $\mathrm{Sassi}^{4} *$, Imen Ghanmi ${ }^{4}$, Laurent Michely ${ }^{2}$, Youssef Snoussi ${ }^{3}$, Alexandre Chevillot ${ }^{3}$, Stéphanie Lau-Truong ${ }^{3}$, Mohamed M. Chehimi ${ }^{2,3, *}$

1 Photochemistry Department, National Research Centre, Dokki, Giza 12622, Egypt

2 Université Paris Est, CNRS, ICMPE (UMR 7182), 94320 Thiais, France

3 Université de Paris, CNRS, ITODYS (UMR 7086), 75013 Paris, France

4 Faculté des Sciences, Unité de Recherche Électrochimie, Matériaux et Environnement UREME

(UR17ES45), Université de Gabès, 6000, Gabès, Tunisia

* Correspondence: akhali175@yahoo.com (AMK); wafa.sassi@issatgb.u-gabes.tn (WS);

mohamed.chehimi@cnrs.fr ; mmchehimi@yahoo.fr (M.M.C.)

\begin{abstract}
:
There is an ever growing interest worldwide in the development of biochar from a large variety of agrowastes. This work contributes to the domain by tackling an agrowaste represented in pomegranate peels powder. The latter was activated by acid treatment and then pyrolyzed to generate low cost biochar. To enrich the surface of the resulting biochar, it was arylated with various in-situ generated diazonium salts of 4-aminobenzoic acid $\left(\mathrm{H}_{2} \mathrm{~N}-\mathrm{C}_{6} \mathrm{H}_{4}-\mathrm{COOH}\right)$, sulfanilic acid $\left(\mathrm{H}_{2} \mathrm{~N}-\mathrm{C}_{6} \mathrm{H}_{4}-\mathrm{SO}_{3} \mathrm{H}\right)$ and Azure A dye. At a certain point, it was important to monitor the effect of diazonium nature and concentration on the arylation process. Surface modification of biochar was characterized with TGA and Raman analyses which showed gradual changes in the arylation of biochar at low concentrations of $10^{-5}, 10^{-4}$ and $10^{-3} \mathrm{M}$ of 4aminobenzoic acid. Interestingly, Azure A diazonium salt induces lower extent of surface modification, likely due to steric hindrance. One key feature of this work is the correlation between D/G Raman peak intensity ratios and the mass loading of the aryl groups. To the very best of our knowledge this is the first report ever on diazonium modification of biochar and opens new avenues for this carbon allotrope, its surface arylation and applications.
\end{abstract}

Keywords: biochar, agrowaste, pomegranate peel, diazonium salts, arylation, surface modification.

\section{Introduction}

Arylation of carbon allotropes is a very well established mean of preparing functional based carbon materials [1] for various applications comprising fillers [2] and reinforced polymeric matrices [3], support of (electro) nanocatalysts [4], flexible gas sensors [5, 6], electrode materials [7], and photovoltaic applications [8]. Although graphene, carbon nanotubes, fullerene are advanced carbon based materials, yet they are excessively expensive which limits the expansion of their utilization. Instead, one 
can take advantage of biochar(s) generated from the pyrolysis of agrowastes and other biomass resources. Biochar can be regarded as a promising candidate possessing several attractive features which can further be tuned via acid or base surface chemical treatment [9], loading of alkali metal oxalate loading [10], amine functionalization using diethanolamine [11], or silanization [12]. However, arlyation with diazonium salts has much to offer due to the commercial availability of several aromatic amine precursors of diazonium compounds with several functional groups ($\mathrm{COOH},-\mathrm{NH}_{2},-\mathrm{SO}_{3} \mathrm{H}$, perfluro alkyl chain, $\mathrm{N}_{3} \equiv \mathrm{C}$ azide, etc...) to control numerous phyico-chemical properties and post reactivity [13].

Diazonium salts are chief surface modifiers and coupling agents as they spontaneously react with carbon allotropes $[1,13]$. This led us to reason that they could be employed to tailor the surface modification of biochar.

In this work, we bridge the best of two concepts: facile surface modification of carbon allotropes with diazonium salts and pyrolysis process to fabricate biochar from agrowaste (pomegranate peel powder). These materials derived from wastes have not been subjected to arylation using diazonium salts, hence the interest of this investigation. To the very best of our knowledge, no biochar designed by slow pyrolysis has been to surface modification with aryl diazonium salts. On the scientific viewpoint we feature biochar modification in a facile way, at room temperature for multiple purposes. On the technological viewpoint, biochar have much to offer as functional materials when equipped with reactive aryl groups.

Subsequently, we describe the preparation procedure of biochar pyrolysis to pomegranate peels. These carbon allotropes were post functionalized with aryl diazonium salts comprising in-situ generated diazonium salts from 4- amino benzoic acid, sulfanilic acid and Azure A. Pristine and modified biochar materials were characterized by X-ray diffraction, thermal gravimetric analysis (TGA) and Raman spectroscopy.

\section{Experimental}

\subsection{Chemicals}

4-aminobenzoic acid, sulfanilic acid, $\mathrm{NaNO}_{2}$ and $\mathrm{HCl} 37 \%$ were Aldrich products. Azure A was supplied by Alfa Aeser.

\subsection{Biochar preparation}


Pomegranate fruits were collected from the region of Kettana (Gabès region, Tunisia). Biochar was prepared by pyrolysis of pomegranate peel dry powder. The peels were washed thoroughly until the washings were clear. The peels were then dried in an oven at $100{ }^{\circ} \mathrm{C}$ for $24 \mathrm{~h}$. The final dried peels were ground and pyrolyzed under nitrogen. $10 \mathrm{~g}$ of dried peel powder was heated with a temperature ramp of $1{ }^{\circ} \mathrm{C} / \mathrm{min}$; the final temperature was set $1000{ }^{\circ} \mathrm{C}$. The biochar was left then to cool down. The biochar yield was about $21 \%$. The biochar was then acid-treated with $\mathrm{H}_{3} \mathrm{PO}_{4}(40 \%)$ at $105^{\circ} \mathrm{C}$ for $4 \mathrm{~h}$.

\subsection{Arylation of Biochar}

$200 \mathrm{mg}$ of biochar were dispersed in $50 \mathrm{~mL}$ of deionized water under sonication for 10 min then kept in ice-water bath with maintaining the temperature in the range of $0-5{ }^{\circ} \mathrm{C}$. For this dispersion, a 4-aminobenzoic acid solution was supplemented. The latter was prepared by dissolving a proper amount of 4aminobenzoic in $5 \mathrm{~mL}$ of $37 \% \mathrm{HCl}$ to have $\left(10^{-3} \mathrm{M}\right)$ concentration. The mixture was then maintained in an ice-water bath. An aqueous solution of $\mathrm{NaNO}_{2}$ was then added with retaining the ratio of $1: 1$ between 4-aminobenzoic acid and $\mathrm{NaNO}_{2}$. The reaction was pursued with stirring for $60 \mathrm{~min}$. Thereafter, the suspension was centrifuged and washed several times with distilled water to remove any residues then dried.

The steps were repeated to synthesize arylated biochar-pomegranate with lower concentrations of aryl diazonium salt $\left(10^{-4} \mathrm{M}\right.$ and $\left.10^{-5} \mathrm{M}\right)$ taking in consideration a fixed ratio of 1:1 between 4-aminobenzoic acid and $\mathrm{NaNO}_{2}$.

The same arylation procedure to biochar was pursued by utilizing sulfanilic acid and Azure A as diazonium salts precursors at $\left(10^{-3} \mathrm{M}\right)$ instead of 4-aminobenzoic acid.

\subsection{Characterization of biochar samples}

An X'Pert-Pro Panalytical diffractometer was used to record diffraction pattern of the untreated biochar. A cobalt X-ray source $(\lambda=1.7889 \AA)$ was used and the analysis was performed with Bragg-Brentano geometry.

Pristine and arylated biochar samples were characterized through Raman technique by using Horiba Labram HR Evolution machine fitted with a $\mathrm{He}-\mathrm{Ne}$ laser beam the wavelength of which was set at $514 \mathrm{~nm}$. 
TGA measurements were performed with a Setaram apparatus (Setsys Evolution model). The ramp was from RT to $800{ }^{\circ} \mathrm{C}$ at $10{ }^{\circ} \mathrm{C} / \mathrm{min}$ heat rate under argon.

\section{Results and Discussion}

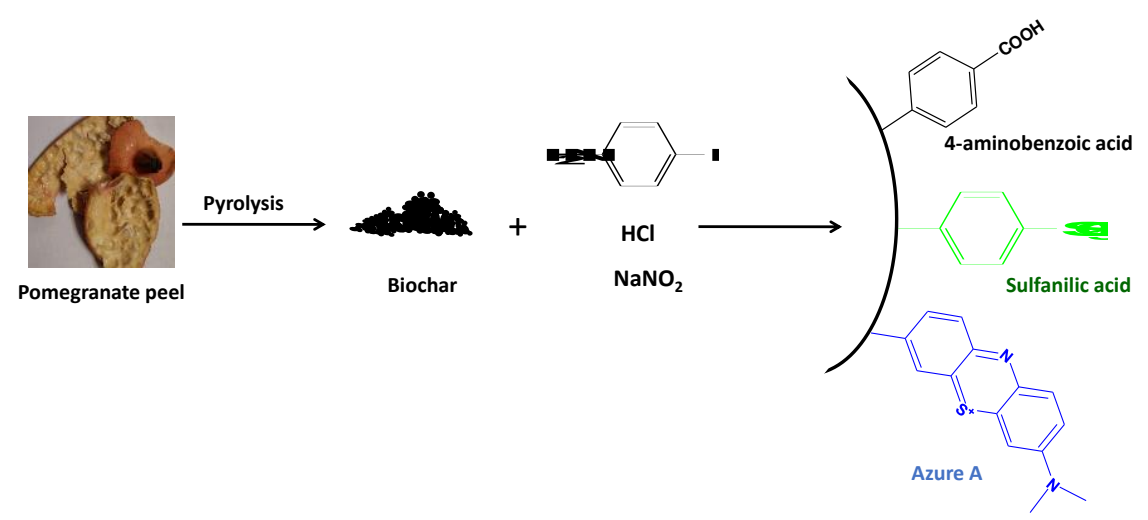

Figure 1. Schematic illustration of the preparation of arylated biochar from pomegranate peel agrowaste.

Figure 1 shows the pathway for the making of arylated biochar starting from pomegranate peel agrowaste. Characterizing arylated pomegranate-based biochar with different diazonium salts were followed up with monitoring different concentrations of 4-amino benzoic acid. We compared dizonium salts of 4-amino benzoicm sulfanilic acid and Azure A at certain concentration $\left(10^{-3} \mathrm{M}\right)$.

The XRD patterns of pomegranate based biochars pyrolyzed at $1000{ }^{\circ} \mathrm{C}$ is shown in Figure 2. Crystalline and semi-crystalline phases exist in biochar. Raising the pyrolytic temperature boosts volatilizing the organic compounds. Hence, it allows generating more pores. XRD spectra demonstrate the destruction of the crystalline structure. Some shifts in biochar XRD peaks with altering their intensities may arise according to the pyrolysis temperature. Relatively broad peaks appear at $(2 \theta)=24.6^{\circ}$ and $44^{\circ}$. 


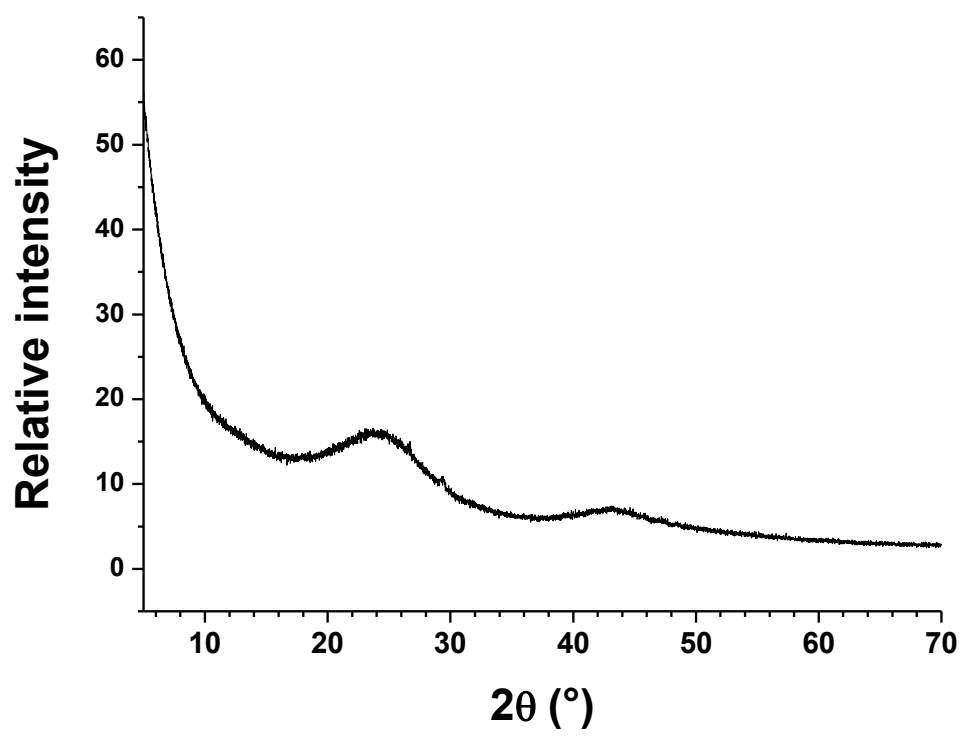

Figure 2. XRD of pomegranate peel biochar.

Thermogravimetric analysis (TGA) was employed to investigate the thermal stability of the biochar upon being functionalized with various concentrations of aryl diazonium salts as shown in Figure 3a. The precursors of these salts are 4aminobenzoic acid $\left(10^{-5}, 10^{-4}\right.$ and $\left.10^{-3} \mathrm{M}\right)$, sulfanilic acid and Azure $\mathrm{A}\left(10^{-3} \mathrm{M}\right)$. The analysis was carried out in a temperature range up to $800{ }^{\circ} \mathrm{C}$. The pyrolysis temperature has a role in determining the thermal stability of biochar. In Figure 2a, the control sample of pristine biochar showed a steady behavior upon increasing the temperature with maintaining its principal weight of biochar without considerable loss. The start of weight loss is monitored at $420{ }^{\circ} \mathrm{C}$ up to $747^{\circ} \mathrm{C}$. The functionalized samples of biochar exhibited a systematic behavior in thermal stability as the sample showing the highest weight loss is for the functionalized one $\left(10^{-3} \mathrm{M}\right) 4$-aminobenzoic acid. This is interesting to prove that as the concentration of aryl diazonium salt decreases in the sample, it approaches the thermal stability of pristine biochar sample. At relatively low temperature at $50-80{ }^{\circ} \mathrm{C}$, the weight loss can be correlated to liberated adsorbed water. Some variations are observed at higher temperatures due to the degradation of functional groups on the surface of the samples. The samples with aryl diazonium salt show gradual weight loss $180-490{ }^{\circ} \mathrm{C}$ as a result of evolving volatiles. The weight loss in this stage may be ascribed to the decomposition as carbon turns to $\mathrm{CO}, \mathrm{CO}_{2}$ and $\mathrm{CH}_{4}$. The lowest weight loss corresponds to biochar by 
losing $3 \%$ of the original weight varying upon being functionalized by 4aminobenzoic acid. Meanwhile, the highest concentration of the used diazonium salt precursor displays the highest weight loss to reach $14 \%$ of its weight. As the concentration of the latter decreases, lower weight loss takes place approaching the control biochar sample. Above $490{ }^{\circ} \mathrm{C}$, a faster weight loss takes place than the previous stage. Upon comparing the other diazonium salt precursors namely; sulfanilic acid and Azure A, we can notice a similar behavior to the previously investigated samples as shown in Figure $3 \mathrm{~b}$. However, the functionalized biochar with sulfanilic acid shows higher thermal stability up to $584{ }^{\circ} \mathrm{C}$ losing $\sim 12 \%$ of its original weight. On the other hand, the sample loaded with Azure A which loses $\sim 7$ $\%$ at relatively lower temperature $503{ }^{\circ} \mathrm{C}$. 

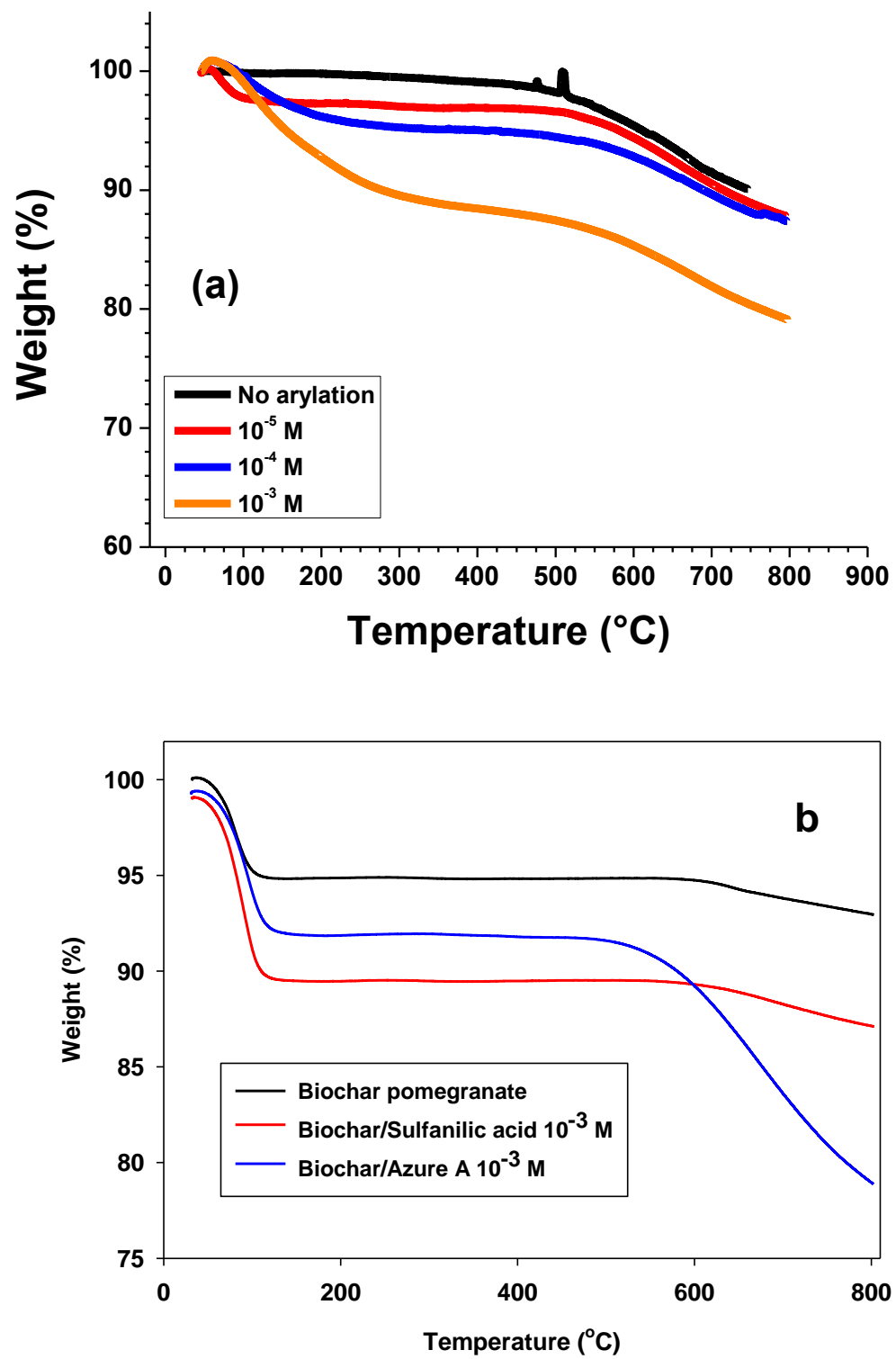

Figure 3. TGA thermograms of biochar loaded with: a) different concentrations of 4- aminobenzoic acid $\left(10^{-3}, 10^{-4}\right.$ and $\left.10^{-5} \mathrm{M}\right)$ b) sulfanilic aicd or Azure $\mathrm{A}\left(10^{-3} \mathrm{M}\right)$.

\subsection{Raman spectroscopy}

Raman spectroscopy was used as a vibrational spectroscopy technique for determining the chemical functionalization and the dissemination of chemical bonds in the investigated biochar samples. Figure 4 illustrates the Raman spectra of the tested biochar samples from 800 to $2000 \mathrm{~cm}^{-1}$. D and $\mathrm{G}$ bands are located at 
$1350 \mathrm{~cm}^{-1}$ and $1580 \mathrm{~cm}^{-1}$ respectively. It is substantial to influence of changing the generated diazonium salts functional groups onto the surface of biochar. D and $G$ bands are designated to the disordered graphite with indicating $\mathrm{sp}^{2}$ carbon atoms in graphitic substrates. The $\mathrm{D} / \mathrm{G}$ peak height and area ratios vary through various samples of pristine or functionalized biochar. The values of peak height ratios increase with arylation regardless the nature of the diazonium compound compared to pristine biochar. Interestingly, Figure 4a qualitatively shows that the relative intensity of the $\mathrm{D}$ band increases with the initial concentration of the 4carboxybenzenediazonium salt, and exceeds unity at high grafting.

From TGA and Raman analyses for the investigated samples, we have derived a stupendous relationship between $\mathrm{D} / \mathrm{G}$ versus weight $\%$. It depends on estimating the residual weight $\%$ of biochar(s) at $400{ }^{\circ} \mathrm{C}$ with respect to $\mathrm{D} / \mathrm{G}$ ratio as shown in Figure 5. The results showed a reasonable relationship upon increasing the functionalization content with diazonium salts onto biochar reaching a plateau region as a sign of saturation of these loadings on biochar surface. The increased mass loading for higher initial concentration of 4-carboxybenzenediazonium salt is due to the oligomerization of the aryl groups $[13,14]$. Since, new aryl radicals attach to those already tethered to the biochar, clearly no further direct modification of biochar could be probed by Raman. This is the reason why at higher mass loading, a steady state D/G intensity ratio is reached. It is noteworthy that diazotized Azure A provides lowest modification of biochar, likely due to steric hindrance which prohibits oligomerization of Azure A units. For this reason, there is less loading and lower D/G change. Elsewhere, it was demonstrated that on multiwalled carbon tubes, arylation with Azure A was found to yield the thinnest aryl layer compared to the diazonium salts of Neutral Red and Congo Red [15]. Yet, the pomegranate biochar modification with $-\mathrm{C}_{6} \mathrm{H}_{4}-\mathrm{COOH}$ groups at initial diazonium concentration of $10^{-5} \mathrm{M}$ far exceeds that of the pristine pomegranate biochar $(\mathrm{D} / \mathrm{G}=0.8)$ or pristine olive pit biochar $(\mathrm{D} / \mathrm{G}$ $=0.66$ [16]) Azure A corresponds to D/G value of To account for the Raman-TGA results, Figure $5 \mathrm{~b}$ schematically illustrates the effect of low, medium and high arylation of biochar on $\mathrm{D} / \mathrm{G}$ peak intensity ratio. 

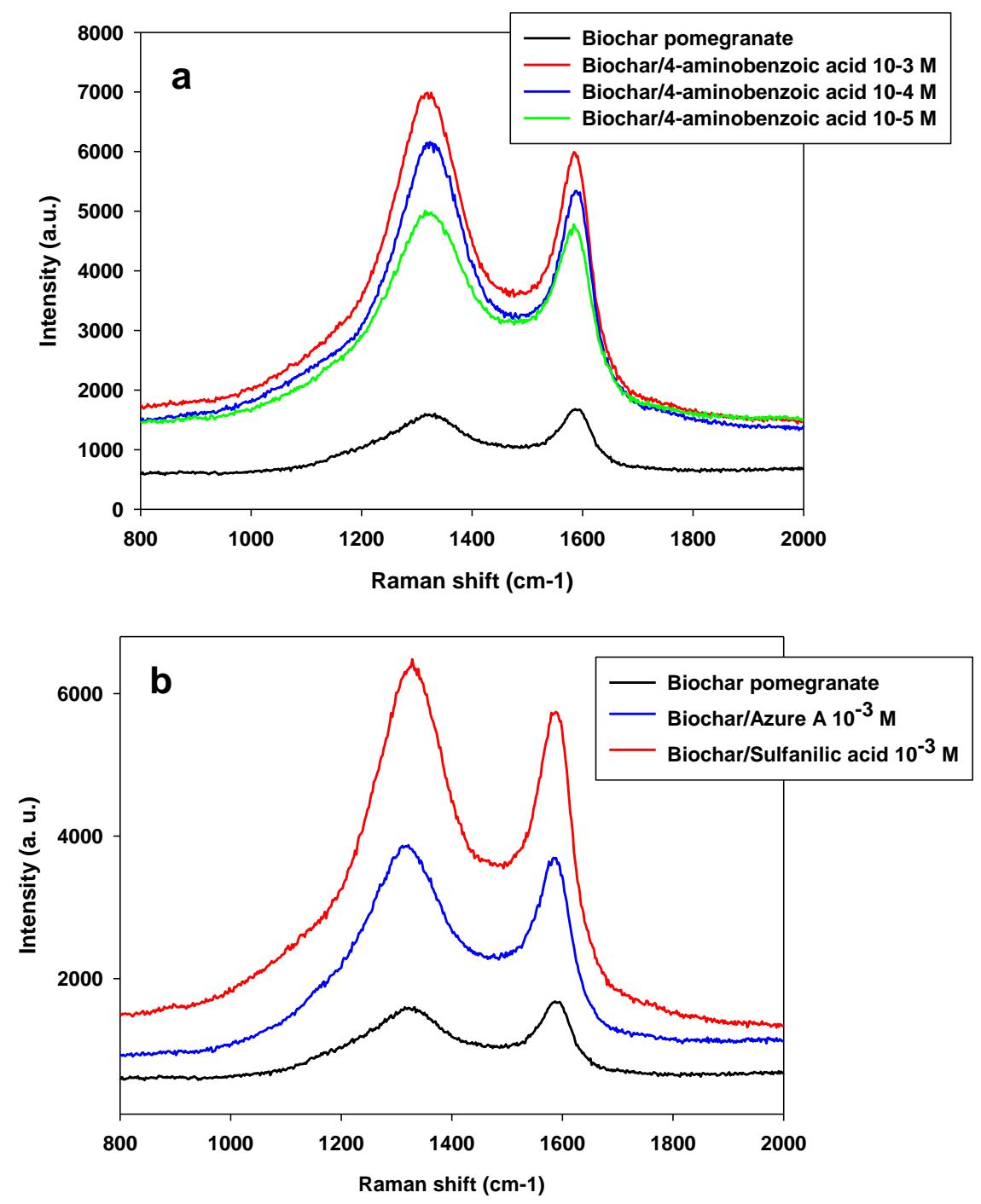

Figure 4. Raman spectra of biochar: a) In the presence of different concentrations of 4- aminobenzoic acid $\left(10^{-3}, 10^{-4}\right.$ and $\left.10^{-5} \mathrm{M}\right)$ b) sulfanilic aicd or Azure $\mathrm{A}\left(10^{-3} \mathrm{M}\right)$. 


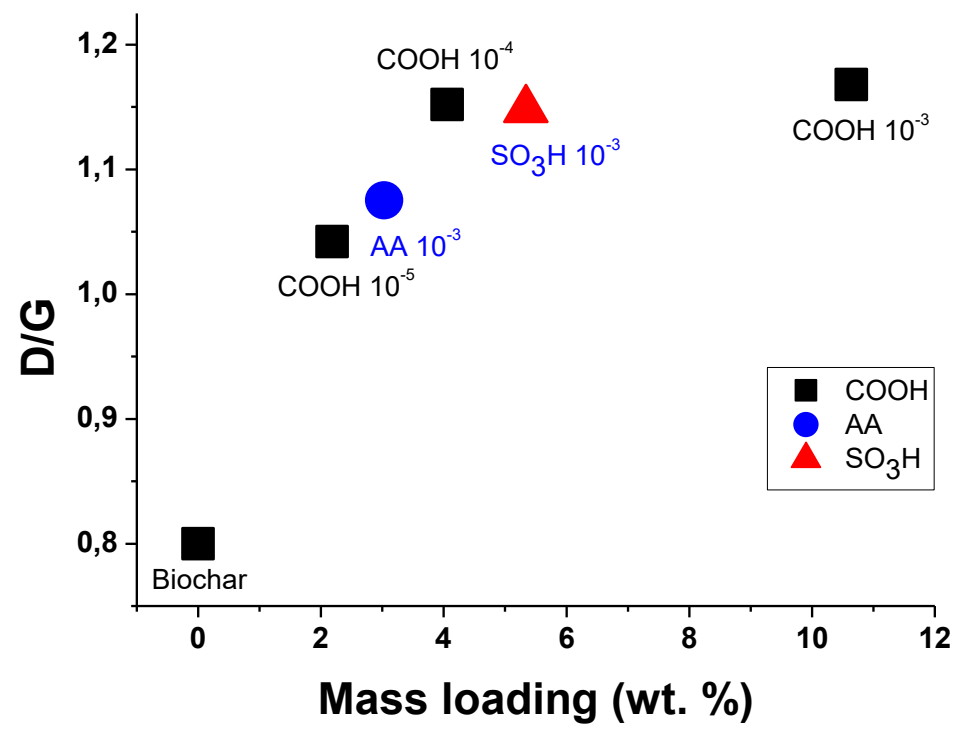

(b)

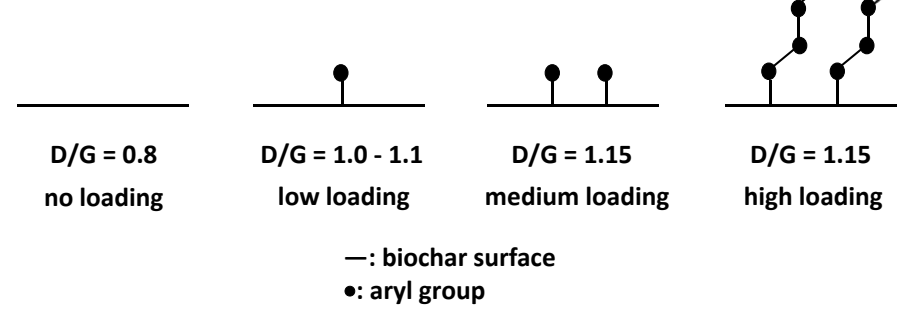

Figure 5. A relationship between $D / G$ and mass loading (wt.\%) for different biochar samples (a), and schematic illustration of stepwise aryl grafting density effect on D/G (b).

\section{Conclusion}

A biochar derived from pomegranate peel powder, as an agrowaste, has been prepared. The preparation step of biochar was carried out by pyrolysis at $1000{ }^{\circ} \mathrm{C}$ followed by functionalization through arylation with $-\mathrm{COOH},-\mathrm{SO}_{3} \mathrm{H}$ and $-\mathrm{N}\left(\mathrm{CH}_{3}\right)_{2}$ diazonium salts. The diazo-precursors of these salts are 4- aminobenzoic acid, sulfanilic acid and Azure A. TGA analysis showed a proportional thermal stability of the investigated biochar samples with respect to the diazonium salts concentration. From the obtained TGA and Raman measurements, we have reached a phenomenal relationship expressing the dependence of $\mathrm{D} / \mathrm{G}$ ratio on the mass loading of biochar to reach a saturation condition at relatively high content of diazo-functionalization at $\left(10^{-3} \mathrm{M}\right)$. The steady state of $\mathrm{D} / \mathrm{G}$ is likely to be due to oligomerization of aryl units. In this situation, there is no direct modification of biochar and thus no more increase in $\mathrm{D} / \mathrm{G}$ value was noted. Interestingly, Azure A diazonium salt imparts lowest D/G 
value compared to other salts (at $10^{-3} \mathrm{M}$ initial concentration) due to steric hindrance and thus limited arylation with bulky molecules.

To the very best of our knowledge, this is the first report on diazonium modification of biochar, prepared by slow pyrolysis of pomegranate peel. This first example can be adapted to any other agrowaste-derived biochar, which is another step towards the valorization of agrowastes.

\section{Acknowledgements}

A.M.K. and M.M.C. would like to thank both the French and Egyptian Governments for funding AMK's contribution through a fellowship granted by the French Embassy in Egypt (Institut Francais d'Egypte) and Science and Technology Development Fund (STDF)-Egypt, Project number (42248).

\section{References:}

[1] C. Gautier, I. Lopez, T. Breton, Materials Advances (2021).

[2] M. Sandomierski, A. Voelkel, Journal of Inorganic and Organometallic Polymers and Materials (2020) 1-21.

[3] A. Bensghaïer, K. Forro, M. Seydou, A. Lamouri, M. Mičušik, M. Omastová, M. Beji, M.M. Chehimi, Vacuum 155 (2018) 178-184.

[4] P. Mirzaei, S. Bastide, A. Aghajani, J. Bourgon, E. Leroy, J. Zhang, Y. Snoussi, A. Bensghaier, O. Hamouma, M.M. Chehimi, Langmuir 35 (2019) 14428-14436.

[5] O. Hamouma, N. Kaur, D. Oukil, A. Mahajan, M.M. Chehimi, Synthetic Metals 258 (2019) 116223.

[6] D. Guettiche, A. Mekki, B. Lilia, T. Fatma-Zohra, A. Boudjellal, Journal of Materials Science: Materials in Electronics 32 (2021) 10662-10677.

[7] D. Hetemi, V. Noël, J. Pinson, Biosensors 10 (2020) 4.

[8] L. Lazzarin, M. Pasini, E. Menna, Molecules 26 (2021) 5286.

[9] D. Zhang, Y. Li, A. Sun, S. Tong, G. Su, X. Jiang, J. Li, W. Han, X. Sun, L. Wang, Science of The Total Environment 694 (2019) 133701.

[10] L. Wang, Y. Shen, Cellulose 28 (2021) 3473-3483.

[11] B. Sajjadi, W.-Y. Chen, D.L. Mattern, N. Hammer, A. Dorris, Journal of Water Process Engineering 34 (2020) 101166.

[12] X. Sun, Y. Qin, B. Wu, J. Li, C. Xue, Environmental Science \& Technology (China) 42 (2019) 68-73.

[13] A.A. Mohamed, Z. Salmi, S.A. Dahoumane, A. Mekki, B. Carbonnier, M.M. Chehimi, Advances in colloid and interface science 225 (2015) 16-36.

[14] D. Belanger, J. Pinson, Chemical Society Reviews 40 (2011) 3995-4048.

[15] A. Bensghaïer, S.p. Lau Truong, M. Seydou, A. Lamouri, E. Leroy, M. Mičušik, K. Forro, M. Beji, J. Pinson, M.r. Omastová, Langmuir 33 (2017) 6677-6690.

[16] A.M. Khalil, L. Michely, R. Pires, S. Bastide, K. Jlassi, S. Ammar, M. Jaziri, M. Chehimi, Applied Sciences 11 (2021) 8513. 